\title{
Projection Structures of DNA-Dps Co-crystals are Determined by the Length of the Incorporated DNA
}

Andrey Moiseenko ${ }^{1}, K_{\text {Ksenia Tereshkina }}^{2}$, Natalia Loiko ${ }^{3}$, Yana Danilova ${ }^{1}$, Yury Krupyansky ${ }^{2}$, Olga S. Sokolova ${ }^{1}$

1. M.V. Lomonosov Moscow State University, Faculty of Biology, Moscow, Russia.

2. N.N. Semenov Institute of Chemical Physics, Russian Academy of Sciences, Moscow, Russia

3. Federal Research Center "Fundamentals of Biotechnology", Russian Academy of Sciences.

Under the influence of adverse environmental conditions, E. coli and other bacteria are able to transit to a stationary phase, which ensures the successful survival of these cells for longer periods of time. The stationary phase is characterized by changing the cellular morphology. Appropriate molecular mechanisms that provide this ability of bacterial cells to survive are currently the subject of active research. One of the universal mechanisms for responding to stress is increasing the synthesis of specific histone-like proteins that bind circular DNA, protecting the nucleoid from stress factors. In Gram-positive and Gram-negative bacteria, such as E. coli, as well as in Archaea, the main DNA-binding protein is Dps. It is overexpressed in the cells undergoing oxidative, temperature, toxic starvation and other types of stresses, and non-specifically binds circular DNA. As a result, two-and three-dimensional crystalline arrays are often observed in the cytoplasm of the starving cells. Under starvation conditions, the amount of Dps can increase to 200,000 molecules in one wild-type E. coli cell.

The crystal structure of Dps was solved previously and revealed that the protein is formed by twelve individual subunits assembled with tetrahedral symmetry. The purified Dps, upon mixing with DNA form a large and highly ordered two-dimensional, arrays in vitro. Here, we studied the structure of such twodimensional DNA-Dps co-crystals using electron microscopy.

Crystals were prepared by mixing purified Dps with DNA (either long circular pBlueScript, or short 24 b.p. linear fragment) by adding a 3 ul drop of Dps solution to the carbon-coated glow-discharged EM grid (SPI) followed immediately by adding a $1 \mathrm{ul}$ drop of DNA. After incubation for $15 \mathrm{sec}$, the excess of liquid was blotted away with filter paper, and grids were stained with a $1 \%$ aquatic solution of uranium acetate for $30 \mathrm{sec}$ and air-dried. The stained grids were analyzed on an analytical transmission electron microscope JEM-2100 (Jeol, Japan) equipped with LaB6 filament with accelerating voltage $200 \mathrm{kV}$ and low-dose conditions. Images that contain crystal lattices (fig. 1A, 2A) were filtered, the peaks were masked and all power spectrum outside peaks were filled with zeroes. Then, inverse Fourier transform results in a filtered image, which is formed with peak signal only (fig. 1C, 2C). Additional steps to improve the resolution included the use of the Fourier filter, CTF correction, lattice distortion correction using estimated lattice parameters. Finally, the data was averaged over a set of images and final projection maps were built (fig. 1D, 2D).

When we compared the projection map of the Dps-DNA(pBlueScript) to the map of the Dps-DNA(24 b.p.) we noticed that Dps particles were oriented differently within the two crystals. Comparison of the Dps crystal structure and its calculated electrostatic potential revealed that pBlueScript DNA may be located in the crystal in the crevices between Dps molecules, while the short 24 b.p. DNA is most likely interacting with the positively charged area on the side of the Dps. Thus the length of the DNA determines the overall orientations of the Dps in the co-crystal [1]. 


\section{References:}

[1] Electron microscopy was done at User Facilities Centers of Moscow State University "Electron microscopy in life sciences" and at Research Resource Center "Molecular and cell technologies" of Sankt-Peterburg State University. Authors acknowledge the financial support from RSF (grant \# 14-2400031)
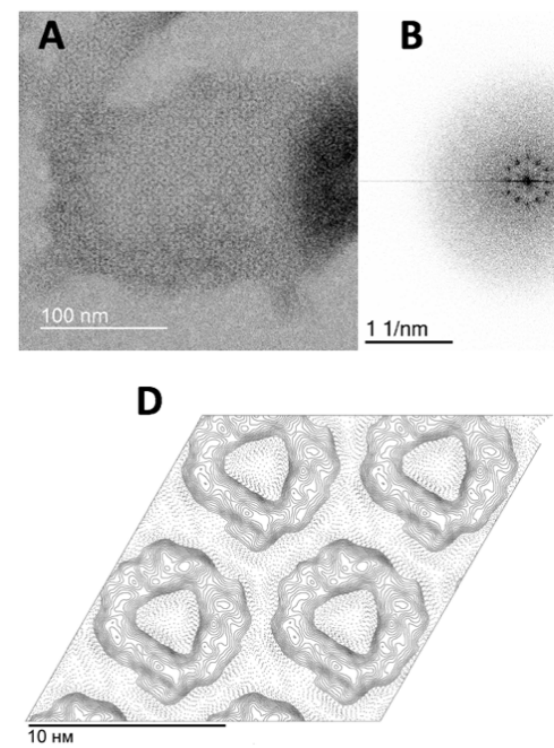

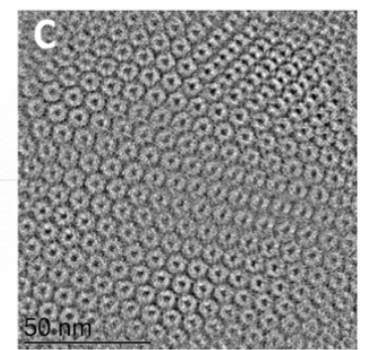

$\mathbf{E}$

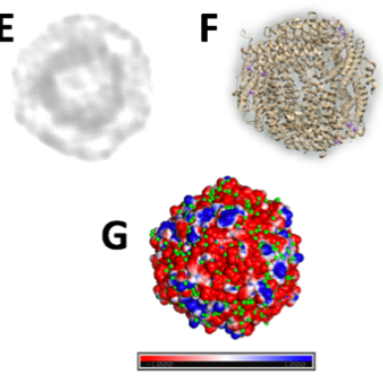

Figure 1. Structure of single-layered Dps crystal in the presence of pBlueScript DNA: (A) TEM of the single-layered crystal; (B) Fourier transform; (C) filtered crystal; (D) projection structure of crystal; (E) projection of the low-pass filtered Dps crystal structure (pdb code 1dps) in the same orientation, as projections in (D); (F) Dps crystal structure in the same orientation, as its projection in (E); (G) electrostatic potential of the Dps crystal structure: red - negative a.a; blue - positive a.a.; green - water molecules.

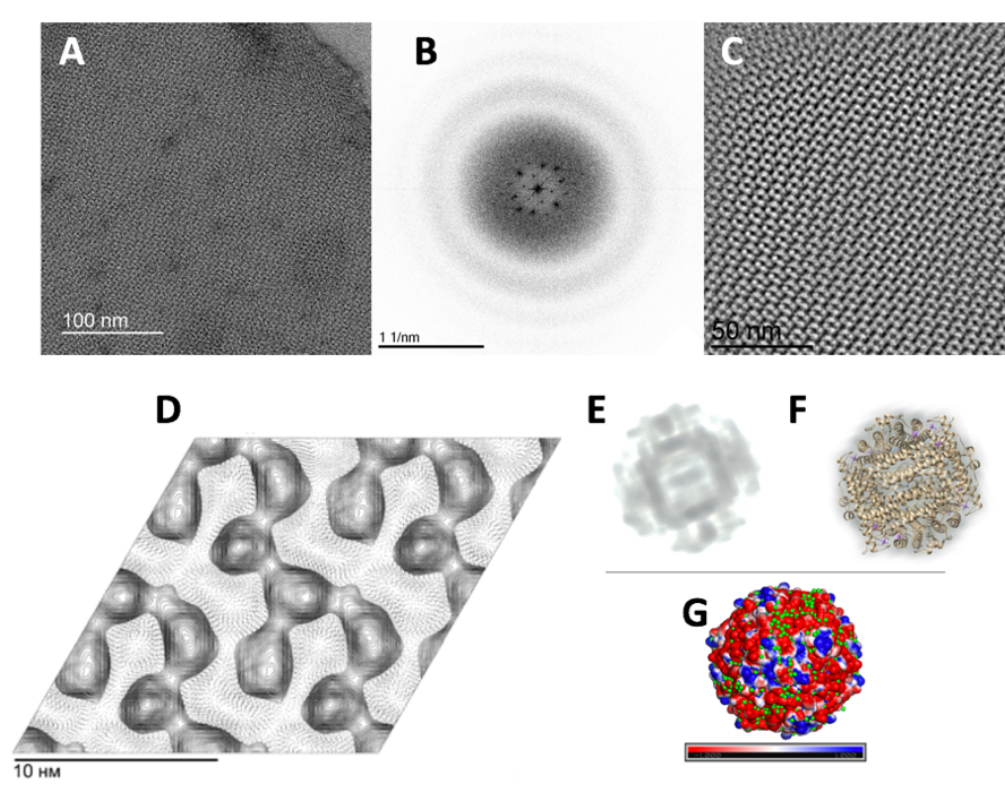

Figure 2. Structure of double-layered Dps crystal in the presence of 24 b.p. DNA: (A) TEM of the doublelayered crystal; (B) Fourier transform; (C) filtered crystal; (D) projection structure of crystal; (E), (F), (G) are designated as corresponding images in Fig. 1. 